\title{
Neutralizing antibodies for the treatment of COVID-19
}

\author{
More clinical trial data are needed to determine whether sera from COVID-19-convalescent patients and \\ neutralizing monoclonal antibodies specific to SARS-CoV-2 antigens can prevent COVID-19 or reduce the severity \\ of the disease in high-risk populations.
}

\section{Shibo Jiang, Xiujuan Zhang, Yang Yang, Peter J. Hotez and Lanying Du}

$\mathrm{N}$ eutralizing antibodies (nAbs) can be passively transferred into individuals before or after viral infection to prevent or treat disease (vaccines, on the other hand, actively stimulate the immune system to produce antigen-specific nAbs to prevent disease). Therapeutic nAbs generally exist in the body for a short time, and their treatment efficacy depends on a variety of factors, including $\mathrm{nAb}$ titre, total amount, specificity and half-life. Neutralizing antibodies with high specificity, strong affinity to target proteins and low toxicity have been used to treat viral infections caused by Ebola virus, cytomegalovirus, influenza virus, human immunodeficiency virus and respiratory syncytial virus. In this Comment, we summarize the neutralizing antibodies, including monoclonal antibodies (mAbs) and nanobodies, for severe acute respiratory syndrome coronavirus 2 (SARS-CoV-2) that are currently in preclinical development and in clinical trials, the antibodies' potential mechanisms of action, and the likely clinical applications of the mAbs and of convalescent sera from patients who have recovered from SARS-CoV-2 infection for the prevention and treatment of coronavirus disease 2019 (COVID-19).

In contrast to SARS-CoV, which was first reported in 2003 and characterized by conditional human-to-human transmission ${ }^{1}$, SARS-CoV-2 has spread exponentially and has led to more than 1.3 million deaths from COVID-19 eleven months after its identification. SARS-CoV-2 and SARS-CoV, which belong to the beta-CoV genera of Coronaviridae, share the same receptor angiotensin-converting enzyme 2 (ACE2) - for viral entry into host cells through the spike (S) proteins on the virus' surface ${ }^{2}$. For both viruses, the $S$ protein consists of S1 and S2 subunits. The receptor-binding domain (RBD) in the $\mathrm{S} 1$ subunit first binds the ACE2 receptor on cells to mediate viral entry via the formation of the RBD-ACE2 complex (Fig. 1a; ref. ${ }^{3}$ ). The S protein then undergoes a conformational change, leading to membrane fusion mediated by the $\mathrm{S} 2$ subunit. The $\mathrm{S}$ protein forms a homotrimer and can undergo spontaneous conformational changes with one or more RBDs, switching from a 'lying down' position to a 'standing up' position to enable receptor binding (Fig. 1b,c; ref. ${ }^{4}$ ). The structural proteins of SARS-CoV-2 namely, $\mathrm{S}$, nucleocapsid $(\mathrm{N})$, membrane $(\mathrm{M})$ and envelope (E) - play pivotal roles in viral infection and replication ${ }^{2}$. Understanding the structure and function of these proteins, particularly of the $S$ protein and its RBD, provides a basis for the rational design and development of SARS-CoV-2-specific $\mathrm{nAbs}$ for the prophylactic prevention and treatment of COVID-19.

There are notable differences between neutralizing mAbs and nanobodies. Monoclonal antibodies and their fragments are normally large, and in order to maintain their conformation and functionality, most have to be produced in mammalian cell expression systems (such as stable cell lines), which usually have a relatively low expression yield and thus high production costs. In contrast, nanobodies are single-domain antibodies with small size, good solubility and strong stability against extreme conditions. They can be easily produced in Escherichia coli and yeast cells at a large scale, resulting in a high expression yield, good functionality and considerably reduced production costs. Owing to their small size, nanobodies typically have higher renal clearance and thus a shorter half-life than mAbs. Yet the half-life of nanobodies can usually be increased by fusing them with long-lived proteins, such as albumin or human fragment crystallizable (Fc) region. Overall, it is important to consider the production cost, stability and half-life of $\mathrm{nAbs}$ in addition to their efficacy and safety.

\section{Preclinical development and clinical trials}

A variety of $n A b s$ against SARS-CoV-2 are in preclinical development, all of which target the S protein (Fig. 2a and Table 1). Most of the identified neutralizing $\mathrm{mAbs}$ are specific to the RBD of the SARS-CoV-2 S protein. Using single $B$ cells from individuals infected with COVID-19, researchers have developed mAbs (P2C-1F11, P2B-2F6, 2-15, 2-7, 1-57, BD-368-2, COV22196, COV2-2130, CC6.29, CC6.30 and CC12.1) that compete with the ACE2 receptor to bind the RBD and neutralize infection in pseudotyped and in authentic SARS-CoV-2 in vitro ${ }^{5-9}$. Based on a naive phage-display single-domain antibody library, human mAbs (n3088 and n3130) screened to bind the RBD of SARS-CoV-2 presented neutralizing activity against pseudotyped and live SARS-CoV-2 infections ${ }^{10}$. Importantly, several neutralizing human mAbs (2-15, BD-3682, COV2-2196, COV2-2130 and CC12.1) showed prophylactic and therapeutic efficacy against SARS-CoV-2 infection in animal models (including human ACE2 (hACE2)-transgenic mice, adenovirushACE2-transduced mice, hamsters and rhesus macaques ${ }^{6-9}$ ). A bivalent $\mathrm{VH}-\mathrm{Fc}$ ab8 $\mathrm{nAb}$ binding to the RBD and its mutants protected both wild-type mice and hamsters against mouse-adapted and authentic SARS-CoV-2 infections ${ }^{11}$.

A number of neutralizing mAbs specific to non-RBD regions of the SARS-CoV-2 $S$ protein are also in preclinical development ${ }^{7,12}$. Human mAbs (4A8, 5-24, 2-17 and 4-8) targeting the $\mathrm{N}$-terminal domain (NTD) exhibited neutralizing activity against pseudotyped and live SARS-CoV-2 infections ${ }^{7,12}$. Other human mAbs (n3072, 2-43 and 2-51) neutralize SARS-CoV-2 infection by binding to the S1 subunit or to undetermined regions on the $S$ trimer ${ }^{7,10}$.

Notably, a few SARS-CoV-2 S-targeting nAbs (mAbs and polyclonal immunoglobulin $\mathrm{G}$ (IgG)) have progressed to clinical trials. SAB-185, a fully human polyclonal $\mathrm{nAb}(\mathrm{hIgG})$ produced via the immunization of genetically engineered 
a
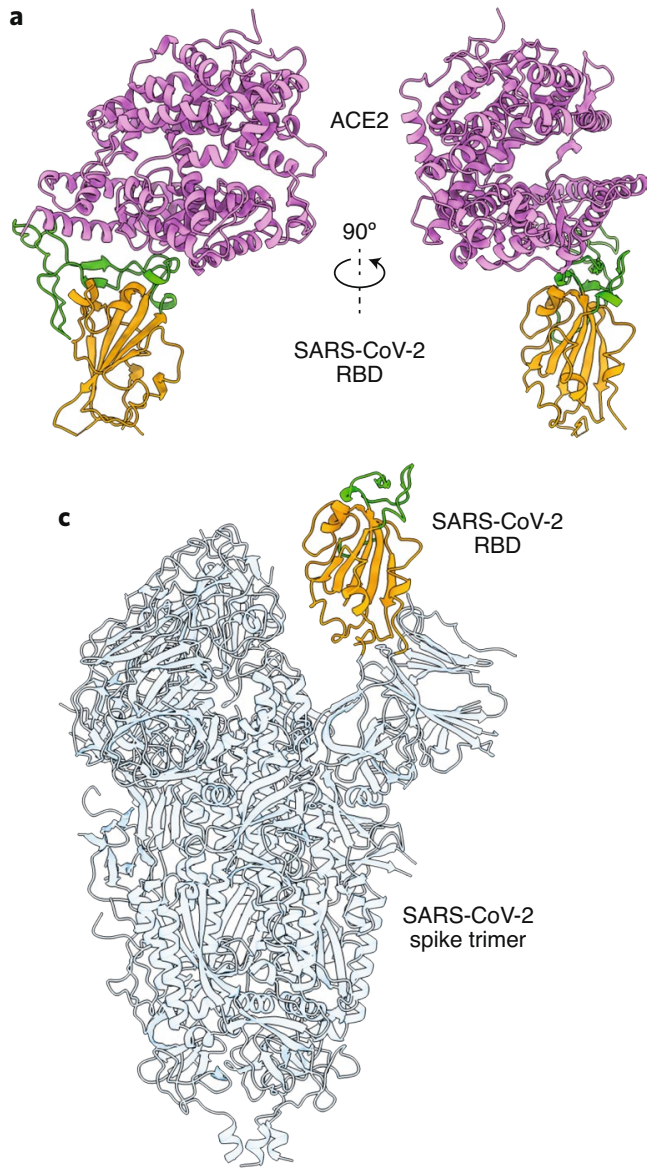

Partially open conformation b

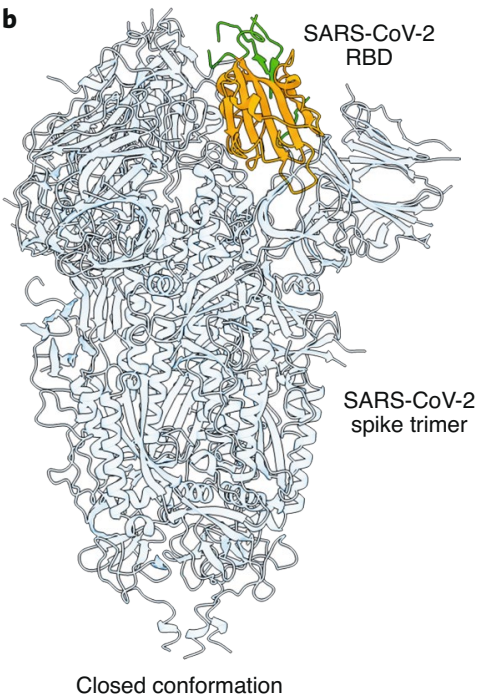

d

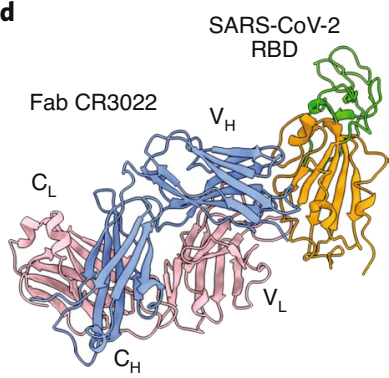

Fig. 1 Structures of the SARS-CoV-2 spike protein and the RBD. a, Front and side views of the crystal structure of the SARS-CoV-2 chimeric RBD in a complex with the hACE2 receptor (PDB 6VW1). The $R B D$ core is depicted in orange, the receptor-binding motif (RBM) in green and $h A C E 2$ in violet. $\mathbf{b}, \mathbf{c}$, Structures of the SARS-CoV-2 S protein trimer in the closed (b; PDB 6VXX) and partially open (c; PDB $6 \mathrm{VYB}$ ) conformations. The RBD core from one $\mathrm{S}$ protomer is depicted in orange and its RBM in green. The rest of the trimeric spike is in light blue. $\mathbf{d}$, Crystal structure of the SARS-CoV-2 RBD in a complex with the human antibody Fab CR3022 (PDB 6W41). The RBD core is depicted in orange and the RBM in green. The light and heavy chains of mAb CR3022 are in pink and blue, respectively.

cattle, is scheduled for a phase $1 \mathrm{~b}$ trial $^{13}$. VIR-7831, a neutralizing human $\mathrm{mAb}$ recognizing a conserved epitope on the $S$ proteins of SARS-CoV-2 and SARS-CoV, has been tested in a phase $2 / 3$ clinical trial for the early treatment of COVID19 in outpatients ${ }^{14}$. Phase 3 trials of a neutralizing human mAb LY-CoV555 and of a neutralizing $\mathrm{mAb}$ combination (REGN10933 + REGN10987) are evaluating their efficacy and safety ${ }^{15}$. The results from the interim analysis of a phase 2 trial of LY-CoV555 indicated that only one dose $(2,800 \mathrm{mg}$ ) of LY-CoV555 showed accelerated natural decline in viral loads over time, whereas the other two doses (7,000 $\mathrm{mg}$ and $700 \mathrm{mg}$ ) did not significantly reduce viral loads by day 11 (ref. ${ }^{16}$ ). On 26
October 2020, the American pharmaceutical company Eli Lilly announced that another trial (the ACTIV-3 trial) of its antibody therapy involving hospitalized patients was stopped because the treatment was ineffective in patients with severe symptoms; all other studies of Bamlanivimab, including ACTIV-2, are still underway ${ }^{17}$. Similarly, the American biotechnology company Regeneron Pharmaceuticals stopped enrolling seriously ill patients with COVID-19 in the clinical trial of REGN-COV2 (combined REGN10933 and REGN10987 mAbs); however, the trial continues to enrol outpatients and hospitalized patients with mild to moderate illness ${ }^{18}$. In November 2020, Bamlanivimab, Casirivimab (REGN10933) and Imdevimab
(REGN10987) were authorized for emergency use by the United States Food and Drug Administration ${ }^{19,20}$.

Neutralizing nanobodies targeting SARS-CoV-2, particularly the RBD, are at preclinical stages of development. By using synthetic or naive llama nanobody phage-display libraries, screened SARS-CoV-2 RBD-targeting nanobodies (IE2, 2F2, 3F11, 4D8, 5F8, H11-D4 and H11-H4) blocked RBD-ACE2 binding, neutralizing pseudotyped and live SARS-CoV-2 infections in vitro ${ }^{21,22}$. The Ty1 nanobody, derived from alpacas, was shown to bind to the RBD and block its binding to the ACE2 receptor, neutralizing pseudotyped SARS-CoV-2 infection ${ }^{23}$. Engineering these nanobodies with human Fc improved their neutralizing ability ${ }^{21-23}$. Several multivalent thermostable nanobodies with ultrahigh neutralizing activity (and with half-maximal inhibitory concentration $\left(\mathrm{IC}_{50}\right.$ ) values as low as $0.058 \mathrm{ng} \mathrm{ml}^{-1}$ ) have been engineered; these can bind to multiple distinct and non-overlapping epitopes in the RBD and may prevent mutational escape ${ }^{24}$. Notably, Nb6 (a class I neutralizing nanobody that binds to the $\mathrm{S}$ protein and the RBD and competes with ACE2) and $\mathrm{Nb} 3$ (a class II neutralizing nanobody binding to the $S$ protein but not to the $\mathrm{RBD}$ alone) were identified by screening a yeast surface-display library of synthetic nanobody sequences. Trimeric Nb6 and trimeric $\mathrm{Nb} 3$ strongly inhibited SARS-CoV-2 infection, with $\mathrm{IC}_{50}$ values of $160 \mathrm{pM}$ and $140 \mathrm{nM}$, respectively ${ }^{25}$. These nanobodies have yet to be evaluated in animal models and human trials. In contrast to the full-length IgG, nanobodies can be delivered via aerosols so that they are able to reach the airway epithelia directly.

Neutralizing antibodies targeting RBDs in the $\mathrm{S}$ proteins of SARS-CoV-2, SARS-CoV and the Middle East respiratory syndrome coronavirus (MERS-CoV) can have higher potency than nAbs targeting the NTD and S2 subunit. For instance, cocktail treatments of neutralizing mAbs (such as REGN10933 + REGN10987), which recognize distinct and non-overlapping epitopes on the $\mathrm{RBD}$, may maintain their ability to neutralize mutant strains with $S$ variants, thus preventing or minimizing antibody escape $^{26}$. Because RBD residues are more divergent than those in other regions of the $S$ protein, more $n A b s$ should be developed to target other epitopes in the conserved regions of SARS-CoV-2 or of other surface proteins, so as to improve the antibodies' broad-spectrum activity and efficacy.

Some SARS-CoV-targeting nAbs can also neutralize SARS-CoV-2 infection. 
a

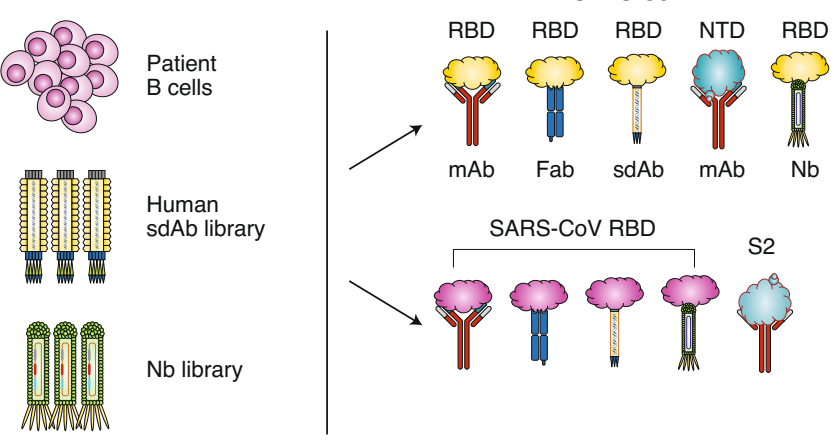

b

(i) In the absence of nAbs

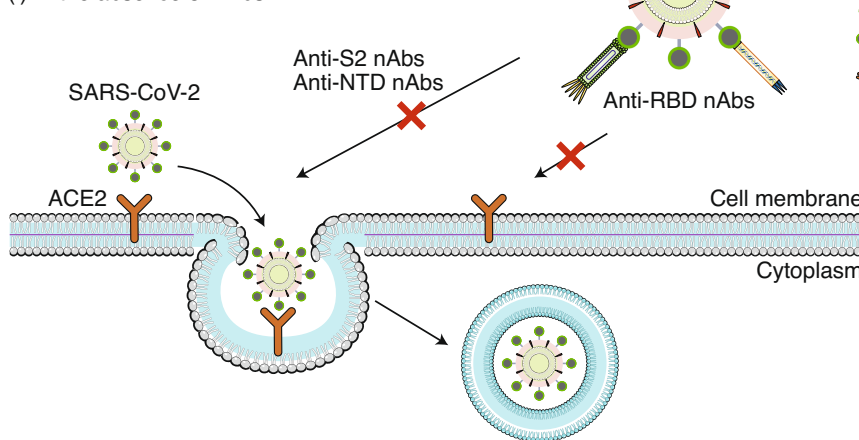

Fig. 2 | Generation of SARS-CoV-2 neutralizing antibodies, and potential mechanisms of action. a, SARS-CoV-2 nAbs may be isolated from patients' B cells, a library of human single-domain antibodies (sdAbs), or a library of nanobodies (Nbs). Different regions of the SARS-CoV-2 S protein are targeted by nAbs, including the RBD and NTD in the S1 subunit. SARS-CoV nAbs with cross-neutralization activity against SARS-CoV-2 may cross-react with the SARS-CoV-2 RBD or S2 subunit. Convalescent plasma from patients infected with SARS-CoV-2 could be used for the treatment of COVID-19. b. Potential mechanisms of action. (i) In the absence of nAbs, SARS-CoV-2 binds to the viral ACE2 receptor via the $R B D$, mediating viral entry into target cells. (ii) In the presence of RBD-specific $n A b s$, the antibodies bind to the RBD and inhibit RBD binding to ACE2, resulting in the inhibition of membrane fusion and the entry of the virus into the host cell. Some non-RBD-targeting nAbs may bind to the NTD, the S trimer or the S2 subunit (thus preventing conformational changes of $\mathrm{S}$ or inhibiting membrane fusion and viral entry). (iii) In the presence of nAbs with suboptimal or negligible neutralizing activity, the antibody-bound virions may enter cells (such as monocytes or macrophages) through the Fc $\gamma \mathrm{R}$, leading to enhanced viral entry, viral replication or inflammation.

Notably, several SARS-CoV-specific nAbs (such as the CR3022 and S303 mAbs, or their functional antibody-binding fragments (Fabs)) bind the SARS-CoV-2 $\mathrm{RBD}$, but not the receptor-binding motif, and thus fail to cross-neutralize SARS-CoV-2 infection ${ }^{27,28}$ (Fig. 1d). Conversely, other SARS-CoV-targeting nAbs (such as S304, S309 and S315, which were isolated from patients infected with SARS-CoV) bind the SARS-CoV-2 RBD by recognizing conserved epitopes, and then cross-neutralize pseudotyped and live SARS-CoV-2 infections ${ }^{27}$. Nonetheless, there is inconsistency in the ability of SARS-CoV-specific nAbs to cross-neutralize SARS-CoV-2 compared with their efficacy in neutralizing SARS-CoV. Therefore, repurposing SARS-CoV nAbs to treat SARS-CoV-2 infection will require protein engineering to improve neutralizing activity by enhancing the antibodies' binding affinity to the viral proteins or the ACE2 receptor. Alternatively, SARS-CoV nAbs with low cross-neutralizing activity against SARS-CoV-2 may be used in combination with SARS-CoV-2 nAbs targeting the SARS-CoV-2 RBD or other epitopes in the S protein to achieve a synergistic neutralizing effect. Such an approach might also be useful for the development of pan-CoV $\mathrm{nAb}$ therapies.

Critically ill patients with COVID19 often present elevated levels of pro-inflammatory cytokines, such as interleukin-6 (IL-6), that are positively correlated with increased viral loads ${ }^{29}$. Also, there is evidence that both lung pathology and immunopathology following the administration of some SARS-CoV vaccines in animals may be related to Th17 host responses ${ }^{30}$. Tocilizumab, an anti-human IL-6 receptor $m A b$, was used to treat COVID-19-related respiratory failure in a patient ${ }^{31}$; after two doses of intravenously injected Tocilizumab, the patient showed improved clinical symptoms, had reduced pulmonary infiltrations and ultimately recovered from COVID-19. Tocilizumab is currently under efficacy and safety trials for the treatment of patients with severe COVID-19 pneumonia ${ }^{32,33}$.

Plasma from convalescent individuals infected with SARS-CoV-2 is being administered to severely ill patients to neutralize viraemia and to counteract cytokine storms (Fig. 2a and Table 1). Transfusion of convalescent plasma containing SARS-CoV-2-specific IgG and nAbs to critically ill patients results in reduced or undetectable viral loads, and relieved or resolved acute respiratory distress syndrome ${ }^{34}$. In addition, patients who were transfused within 44 hours of hospitalization with convalescent plasma containing high-titre anti-RBD IgG antibodies had significantly decreased mortality ${ }^{35}$. Other trials for treating COVID-19 using convalescent human plasma are ongoing ${ }^{36,37}$. In the United States, an analysis of 20,000 patients with COVID-19 found that convalescent plasma therapy is safe and may reduce mortality ${ }^{38}$. Although marginally successful, many factors - such as a recipient's age and comorbidities, and the $\mathrm{nAb}$ titre of the transferred plasma - may affect outcomes. Most recently, the results from an open-label, parallel-arm, phase II multicentre randomized controlled trial (the PLACID trial) conducted on 464 patients with confirmed, moderate COVID19 in 39 public and private hospitals across India have shown that convalescent plasma is not associated with a reduced progression to severe COVID-19 or all-cause mortality, suggesting that the convalescent plasma therapy is ineffective for COVID-19 (ref. ${ }^{39}$ ). On the basis of these results, the following recommendations for future clinical trials of convalescent plasma therapy have been put forward: 
Table 1 | Representative SARS-CoV-2 nAbs in preclinical development and in clinical trials

\begin{tabular}{|c|c|c|c|c|c|}
\hline nAbs & Name & Target antigen & Mechanisms of inhibition or neutralization & $\begin{array}{l}\text { In clinical } \\
\text { trials }\end{array}$ & Reference \\
\hline Human mAbs & P2C-1F11 and P2B-2F6 & SARS-CoV-2 RBD & $\begin{array}{l}\text { Competed with ACE2 to bind RBD, } \\
\text { neutralizing pseudotyped and live } \\
\text { SARS-CoV-2 infections }\end{array}$ & N/A & 5 \\
\hline Human mAbs & $\begin{array}{l}\text { CC6.29, CC6.30 and } \\
\text { CC12.1 }\end{array}$ & SARS-CoV-2 RBD & $\begin{array}{l}\text { Neutralized pseudotyped and live } \\
\text { SARS-CoV-2 infections, protecting hamsters } \\
\text { against SARS-CoV- } 2 \text { challenge (for } C \text { C12.1) }\end{array}$ & N/A & 6 \\
\hline Human sdAbs & n3088 and n3130 & SARS-CoV-2 RBD & $\begin{array}{l}\text { Neutralized pseudotyped and live } \\
\text { SARS-CoV-2 infections }\end{array}$ & N/A & 10 \\
\hline Human mAb & $\mathrm{VH}_{\mathrm{H}} \mathrm{Fc}$ ab8 & SARS-CoV-2 RBD & $\begin{array}{l}\text { Competed with ACE2 to bind RBD, } \\
\text { neutralized pseudotyped and live } \\
\text { SARS-CoV-2 infections, and protected } \\
\text { wild-type mice against mouse-adapted } \\
\text { SARS-CoV-2, or hamsters against } \\
\text { SARS-CoV-2 challenge }\end{array}$ & N/A & 11 \\
\hline Human mAbs & $4 A 8,5-24,2-17$ and $4-8$ & SARS-CoV-2 NTD & $\begin{array}{l}\text { Neutralized pseudotyped and live } \\
\text { SARS-CoV-2 infections }\end{array}$ & N/A & 7,12 \\
\hline Human mAbs & $2-43$ and $2-51$ & SARS-CoV-2 S & $\begin{array}{l}\text { Bound to } S \text { trimer, and neutralized } \\
\text { pseudotyped and live SARS-CoV-2 infections }\end{array}$ & N/A & 7 \\
\hline hlgG Ab & SAB-185 & SARS-CoV-2 S & Neutralized live SARS-CoV-2 infection & Yes & 13 \\
\hline Human $\mathrm{mAb}$ & VIR-7831 & $\begin{array}{l}\text { SARS-CoV-2 and } \\
\text { SARS-CoV S }\end{array}$ & $\begin{array}{l}\text { Recognized a conserved epitope on the } S \\
\text { proteins of SARS-CoV and SARS-CoV-2, and } \\
\text { neutralized live SARS-CoV-2 infection }\end{array}$ & Yes & 14 \\
\hline Human $\mathrm{mAb}$ & LY-CoV555 & SARS-CoV-2 S & $\begin{array}{l}\text { Blocked viral attachment and entry into } \\
\text { human cells, neutralizing SARS-CoV-2 } \\
\text { infection }\end{array}$ & Yes & 15 \\
\hline Human mAb cocktail & $\begin{array}{l}\text { REGN-COV-2 } \\
(\text { REGN10933 + } \\
\text { REGN10987) }\end{array}$ & SARS-CoV-2 RBD & $\begin{array}{l}\text { Blocked RBD-ACE2 binding, neutralizing } \\
\text { SARS-CoV-2 with variant S mutants }\end{array}$ & Yes & 15 \\
\hline Llama-derived nanobodies & $\mathrm{H} 11-\mathrm{D} 4$ and $\mathrm{H} 11-\mathrm{H} 4$ & SARS-CoV-2 RBD & $\begin{array}{l}\text { Blocked RBD-ACE2 binding, neutralizing live } \\
\text { SARS-CoV-2 infection }\end{array}$ & N/A & 21 \\
\hline Humanized nanobodies & $\begin{array}{l}\text { IE2, 2F2, 3F11, 4D8 and } \\
5 F 8\end{array}$ & SARS-CoV-2 RBD & $\begin{array}{l}\text { Blocked RBD-ACE2 binding, neutralizing } \\
\text { pseudotyped and live SARS-CoV-2 infections }\end{array}$ & N/A & 22 \\
\hline Alpaca-derived nanobody & Ty1 & SARS-CoV-2 RBD & $\begin{array}{l}\text { Bound to RBD in up-and-down conformation } \\
\text { and blocked RBD-ACE2 binding, neutralizing } \\
\text { pseudotyped SARS-CoV-2 infection }\end{array}$ & N/A & 23 \\
\hline Human mAbs or Fabs & S304, S309 and S315 & SARS-CoV RBD & $\begin{array}{l}\text { Bound to SARS-CoV-2 RBD, but did } \\
\text { not compete with RBD-ACE2 binding, } \\
\text { neutralizing pseudotyped and live } \\
\text { SARS-CoV-2 infections }\end{array}$ & N/A & 27 \\
\hline $\lg G A b$ & Convalescent plasma & SARS-CoV-2 & $\begin{array}{l}\text { Maintained neutralizing activity against } \\
\text { SARS-CoV-2 infection }\end{array}$ & $\begin{array}{l}\text { Yes (for } \\
\text { treating } \\
\text { patients with } \\
\text { COVID-19) }\end{array}$ & 34 \\
\hline
\end{tabular}

N/A, information not available.

the potential harms of the non-immune components in the convalescent plasma, particularly pro-thrombotic risks, should be evaluated; only convalescent plasma with detectable titres of nAbs should be used; double-blind designs with sham procedure controls should be included; and non-immune plasma should not be used as a control intervention ${ }^{40}$.

\section{Potential mechanisms of action}

The potential mechanisms of action of nAbs for the prevention and treatment of SARS-CoV-2 infection are described in Fig. 2b. In the absence of specific antibodies, SARS-CoV-2 binds the ACE2 receptor through the $\mathrm{RBD}$ in the S1 subunit, mediating viral entry and subsequent membrane fusion. In the presence of $\mathrm{nAbs}$ targeting and binding to the SARS-CoV-2 (or SARS-CoV) RBD, interaction with the ACE2 receptor is blocked and membrane fusion is inhibited. However, several non-RBD-targeting nAbs - or RBD-targeting nAbs that do not compete with the ACE2 receptor - recognize epitopes distinct from the ACE2-binding motif or the non-RBD 
regions of the $\mathrm{S}$ protein. These antibodies have virus-neutralizing properties. They may bind the NTD in the S1 subunit or the $S$ trimer to inhibit conformational changes of the S protein, or may bind S2 to inhibit membrane fusion and subsequent viral entry. In other cases, nAbs (such as S309) may bind cells containing the Fc $\gamma$ receptor (FcyR), leading to antibody-dependent cell cytotoxicity (mediated by natural killer cells) or to antibody-dependent cellular phagocytosis ${ }^{27}$.

However, the presence of antibodies without neutralizing activity (non-nAbs) or with suboptimal neutralizing activity may bear risks. For instance, nAbs that fail to block or neutralize the virus and inhibit its binding with the viral receptor can interact with the Fc $\gamma \mathrm{R}$ to enter target cells, potentially causing antibody-dependent enhancement (ADE). In addition, these antibodies may also bind Fc $\gamma$ R-expressing cells, such as macrophages and monocytes, causing disease enhancement or increased levels of inflammatory cytokines. In some cases, particularly when the $\mathrm{nAb}$ titre is suboptimal, the antibody and the Fc $\gamma \mathrm{R}$ can form an antibody-Fc $\gamma$ R complex that functionally mimics viral-receptor-mediated entry, also leading to ADE, as was shown for anti-SARS-CoV and anti-MERS-CoV nAbs ${ }^{41}$.

For SARS-CoV, a full-length S-protein trimer induced Fc $\gamma \mathrm{R}$-dependent and ACE2-independent ADE in vitro ${ }^{2,42,43}$. In ferret models challenged with SARS-CoV, a recombinant modified vaccinia Ankara-based SARS vaccine expressing the full-length $S$ protein failed to protect ferrets against SARS-CoV infection, resulting in potently increased inflammatory responses with enhanced hepatitis ${ }^{44,45}$. Currently, there is no direct evidence of the occurrence of ADE in patients with SARS or with COVID-19 (ref. ${ }^{30}$ ). In experimental studies, SARS-CoV, SARS-CoV-2 and their vaccines induced pro-inflammatory cytokines (such as IL-6) and immunopathology; this has been postulated to occur through Th17 mechanisms, but it is unclear whether they occur through $\mathrm{ADE}^{30}$. It seems that enhanced immunopathology in animals immunized with a SARS-CoV vaccine following viral challenge may be associated with Th2 immune responses or with eosinophil infiltration ${ }^{46,47}$; in fact, Th2 immune responses have been seen in some patients with COVID-19 (ref. ${ }^{48}$ ). However, there are data to support reductions in eosinophilic immunopathology with alum and Th2 inductions after SARS-CoV-2 vaccination $^{49}$. Also, multiple vaccination studies in non-human primates as well as COVID-19 challenge studies have not shown any evidence of ADE, eosinophilic infiltration or immunopathology in the lungs or in other target organs ${ }^{50,51}$. Yet clinical manifestations in critically ill patients ${ }^{29}$ suggest that more work is needed to identify potential $\mathrm{ADE}$ from antibody treatments for SARS-CoV-2.

In addition to preventing or reducing potential ADE, successful antibody therapies against SARS-CoV-2 infection will require improvements in the pharmacokinetic activity and tissue permeability of the nAbs, as well as the determination of optimal titres and dosage.

\section{Potential clinical applications}

Convalescent patient plasma with potent neutralizing activity and no harmful components may eventually prove useful for treating patients with COVID-19. It will be critical to correlate $\mathrm{nAb}$ titres with the titres of RBD-specific or S1-specific antibodies in order to identify plasma with highly effective and safe nAbs. Also, patients with comorbidities and convalescent plasma that may lead to $\mathrm{ADE}^{52}$ should be excluded for plasma transfusion to reduce the risk of potential adverse effects. Although the United States Food and Drug Administration has issued an emergency use authorization for the application of convalescent plasma to treat hospitalized patients with COVID-19, more randomized clinical trials are needed to determine the efficacy and safety of convalescent plasma ${ }^{53}$.

The application of preclinically tested nAbs to patients with COVID-19 requires intensive study and testing. Yet not all developed SARS-CoV-2-specific nAbs have been evaluated for protection against SARS-CoV-2 infection in animal challenge models, and only a select few are in clinical trials. More clinical trial data are needed. The hope is that antibody cocktails based on nAbs specific to different neutralizing epitopes in the SARS-CoV-2 RBD, or to other regions in the $\mathrm{S}$ protein of SARS-CoV-2, make effective COVID-19 treatments and provide short-term protection against viral infection. If so, the combination of the early administration of $\mathrm{nAbs}$ followed by steroids such as dexamethasone at a later disease stage (to reduce the risk and severity of acute respiratory distress syndrome) may prevent high viral loads and severe pneumonia, and thus save lives. Unlike vaccines, which usually take several weeks to induce antibody production in immunized individuals ${ }^{54}$, neutralizing mAbs may provide immediate protection against viral infection, and thus be suitable for people at all ages and be particularly suitable for high-risk populations and immunocompromised individuals who typically do not generate sufficient $\mathrm{nAbs}$ after vaccination. However, producing effective neutralizing $\mathrm{mAbs}$ in a cost-effective manner, and at scale, is challenging; most mAbs are produced in mammalian cells with relatively low productivity and thus high production costs. Manufacturing approaches that produce effective $\mathrm{nAbs}$ or nanobodies with high expression yield at low cost are acutely needed.

Shibo Jiang ${ }^{1,2}$, Xiujuan Zhang', Yang Yang ${ }^{3,4}$, Peter J. Hotez ${ }^{5}$ and Lanying Du ${ }^{1} \bowtie$

${ }^{1}$ Lindsley F. Kimball Research Institute, New York Blood Center, New York, NY, USA. ${ }^{2}$ Key Laboratory of Medical Molecular Virology (MOE/NHC/CAMS), School of Basic Medical Sciences, Fudan University, Shanghai, China. ${ }^{3}$ Howard Hughes Medical Institute, Yale University, New Haven, CT, USA. ${ }^{4}$ Department of Molecular, Cellular and Developmental Biology, Yale University, New Haven, CT, USA. ${ }^{5}$ Departments of Pediatrics and Molecular Virology and Microbiology, National School of Tropical Medicine, Baylor College of Medicine, Houston, TX, USA.

$凶_{\mathcal{e}-\text { mail: } l d u @ n y b c . o r g}$

Published online: 8 December 2020 https://doi.org/10.1038/s41551-020-00660-2

References

1. Du, L. et al. Nat. Rev. Microbiol. 7, 226-236 (2009).

2. Wang, N., Shang, J., Jiang, S. \& Du, L. Front. Microbiol. 11, 298 (2020).

3. Shang, J. et al. Nature 581, 221-224 (2020).

4. Walls, A. C. et al. Cell 181, 281-292 (2020).

5. Ju, B. et al. Nature 584, 115-119 (2020).

6. Rogers, T. F. et al. Science 369, 956-963 (2020).

7. Liu, L. et al. Nature 584, 450-456 (2020).

8. Cao, Y. et al. Cell 182, 73-84 (2020)

9. Zost, S. J. et al. Nature 584, 443-449 (2020).

10. Wu, Y. et al. Cell Host Microbe 27, 891-898 (2020).

11. Li, W. et al. Cell 183, 429-441 (2020).

12. Chi, X. et al. Science 369, 650-655 (2020).

13. SAb Biotherapeutics, Inc. Safety, tolerability, and pharmacokinetics of SAB-185 in ambulatory participants with COVID-19. ClinicalTrials.gov https://clinicaltrials.gov/ct2/show/ NCT04469179 (2020).

14. Vir Biotechnology, Inc. VIR-7831 for the early treatment of COVID-19 in outpatients (COMET-ICE). ClinicalTrials.gov https://clinicaltrials.gov/ct2/show/NCT04545060 (2020).

15. Clinical trials of monoclonal antibodies to prevent COVID-19 now enrolling. National Institutes of Health https://www.nih.gov/news-events/news-releases/ clinical-trials-monoclonal-antibodies-prevent-covid19-now-enrolling (10 August 2020).

16. Chen, P. et al. N. Engl. J. Med. https://doi.org/10.1056/ NEJMoa2029849 (2020).

17. Lilly statement regarding NIH's ACTIV-3 clinical trial. Lilly https://www.lilly.com/news/stories/statement-activ3-clinicaltrial-nih-covid19 (26 October 2020).

18. May, B. Regeneron halts enrollment of critically ill patients in a COVID-19 antibody trial. BioSpace https://www.biospace.com/ article/regeneron-halts-enrollment-in-covid-19-trial-followingsafety-signal-in-critically-ill-patients (30 October 2020).

19. FDA authorizes monoclonal antibody for treatment of COVID-19. United States Food and Drug Administration https://www.fda.gov/news-events/press-announcements/ coronavirus-covid-19-update-fda-authorizes-monoclonalantibody-treatment-covid-19 (2020).

20. FDA authorizes monoclonal antibodies for treatment of COVID-19. United States Food and Drug Administration https://www.fda.gov/news-events/press-announcements/ coronavirus-covid-19-update-fda-authorizes-monoclonalantibodies-treatment-covid-19 (2020). 
21. Huo, J. et al. Nat. Struct. Mol. Biol. 27, 846-854 (2020). 22. Chi, X. et al. Nat. Commun. 11, 4528 (2020).

23. Hanke, L. et al. Nat. Commun. 11, 4420 (2020).

24. Xiang, Y. et al. Science https://doi.org/10.1126/science.abe4747 (2020)

25. Schoof, M. et al. Science https://doi.org/10.1126/science.abe3255 (2020)

26. Baum, A. et al. Science 369, 1014-1018 (2020).

27. Pinto, D. et al. Nature 583, 290-295 (2020).

28. Yuan, M. et al. Science 368, 630-633 (2020).

29. Chen, X. et al. Clin. Infect. Dis. 71, 1937-1942 (2020).

30. Hotez, P. J., Corry, D. B. \& Bottazzi, M. E. Nat. Rev. Immunol. 20 347-348 (2020).

31. Michot, J. M. et al. Ann. Oncol. 31, 961-964 (2020).

32. Rilinger, J. et al. Trials 21, 470 (2020).

33. Cotter, A. et al. Trials 21, 758 (2020)

34. Shen, C. et al. JAMA 323, 1582-1589 (2020).

35. Salazar, E. et al. Am. J. Pathol. https://doi.org/10.1016/j. ajpath.2020.10.008 (2020).
36. Eckhardt, C. M. et al. Trials 21, 499 (2020). 37. Liu, S. T. H. et al. Nat. Med. 26, 1708-1713 (2020).

38. Joyner, M. J. et al. Mayo Clin. Proc. 95, 1888-1897 (2020).

39. Agarwal, A. et al. BMJ 371, m3939 (2020).

40. Pathak, E. B. BMJ 371, m4072 (2020).

41. Wan, Y. et al. J. Virol. 94, e02015-19 (2020).

42. Kam, Y. W. et al. Vaccine 25, 729-740 (2007).

43. Jaume, M. et al. Hong Kong Med. J. 18(Suppl. 2), 31-36 (2012).

44. Czub, M., Weingartl, H., Czub, S., He, R. \& Cao, J. Vaccine 23, 2273-2279 (2005)

45. Weingartl, H. et al. J. Virol. 78, 12672-12676 (2004)

46. Bolles, M. et al. J. Virol. 85, 12201-12215 (2011).

47. Tseng, C. T. et al. PLoS ONE 7, e35421 (2012).

48. Roncati, L., Nasillo, V., Lusenti, B. \& Riva, G. Ann. Hematol. 99 , 1419-1420 (2020).

49. Hotez, P. J., Corry, D. B., Strych, U. \& Bottazzi, M. E. Nat. Rev. Immunol. 20, 399-400 (2020).

50. Yu, J. et al. Science 369, 806-811 (2020).

51. Feng, L. et al. Nat. Commun. 11, 4207 (2020).
52. Wu, F. et al. Preprint at medRxiv https://doi.org/10.1101/2020 10.08.20209114 (2020)

53. Investigational COVID-19 convalescent plasma: guidance for industry. United States Food and Drug Administration https://www.fda.gov/regulatory-information/search-fda-guidancedocuments/investigational-covid-19-convalescent-plasma (2020) 54. Su, S., Du, L. \& Jiang, S. Nat. Rev. Microbiol. https://doi. org/10.1038/s41579-020-00462-y (2020).

Acknowledgements

This study was supported by the National Institutes of Health (NIH) grant nos. R01AI157975, R01AI139092 and R56AI140872.

Competing interests

The authors declare no competing interests. 